Boletim Científico do Instituto Agronômico do Estado de S. Paulo

Vol. 31

Campinas, outubro de 1972

N. ${ }^{\circ} 25$

\title{
NOVOS CULTIVARES DE ARROZ PARA CULTURA
} IRRIGADA ( $\left.{ }^{\mathbf{1}}\right)$

Derly Machado de Souza, engenheiro-agrônomo, Seção de Arroz e Cereais de Inverno, Instituto Agronômico, RuTH Dos Santos Garruttr, engenheira-agrônoma, Instituto de Tecnologia de Alimentos, e MARIA AmÉlia Charb, engenheira-tecnóloga, Faculdade de Tecnologia de Alimentos, Universidade de Campinas

\section{SINOPSE}

$\mathrm{Na}$ sua maioria, os cultivares de arroz plantados nos Estados do Brasil-Central são de grãos extralongos; a cariopse mede acima de $7 \mathrm{~mm}$ de comprimento por $2,4 \mathrm{~mm}$ de largura.

O cultivar IR8 não teve aceitação por parte do consumiđor, visto apresentar tipo de grão de qualidade e aspecto inferiores. Novos cultivares IR foram introduzidos e testados comparativamente com os mais cultivados no Estado de São Paulo, que são o IAC-120 e o IAC-435.

Foram avaliadas as características agronômicas e tecnológicas (avaliação sensorial) dos referidos cultivares.

Considerando a sua produção relativa, os cultivares IR665-4-1-1, IR665-4-5-5, IR634-9-6-2 e IR634-32-5-3 mostraram-se superiores ao IAC-120 e ao IAC-435 em mais de $30 \%$.

$\mathrm{Na}$ avaliação sensorial do arroz cru, os cultivares que tiveram a preferência geral foram o IR634-32-5-3 e o IAC-435; o IR634-9-6-2 foi rejeitado ao nível de $0,1 \%$.

Para o arroz cozido, contudo, o IAC-120 e o IR665-4-5-5 foram os mais preferidos, enquanto o IR634-32-5-3 foi rejeitado ao nível de 0,1\%, pela aparência geral, pelas características comestíveis e pela preferência geral, da equipe de degustação.

Tomando-se por base todas as características agronômicas da planta (porte, ciclo, tamanho da cariopse e produção) e tecnológicas do arroz beneficiado (aparência geral e características comestíveis), o IR665-4-5-5 é o cultivar que mais se aproxima das exigências dos agricultores e consumidores dos estados do Brasil-Central.

\section{1 - INTRODUÇÃO}

Os cultivares plantados nos estados centrais do Brasil (10)

( $)$ Recebido para publicaçáo em $10^{\circ}$ de dezembro de 1971. 
são, na sua maioria, de grãos extralongos (2), com cariopse acima de $7 \mathrm{~mm}$ de comprimento $\times 2,4 \mathrm{~mm}$ de largura. Esses tipos constituem a preferência do mercado consumidor.

Os trabalhos de introdução de cultivares e melhoramento desenvolvidos no Instituto Agronômico têm sido realizados no sentido de obter tipos de grãos que se assemelhem ao acima indicado $(\mathbf{8}, \mathbf{9})$.

Examinando as fichas de introdução verifica-se que, apesar do seu bom comportamento agrícola, foram raros os cultivares aceitos pelos produtores para plantio comercial, porém na sua quase totalidade foram utilizados em trabalhos de hibridação.

Por ocasião do lançamento do cultivar IR8, pelo IRRI (6), a Seção de Arroz e Cereais de Inverno recebeu uma pequena amostra de sementes que, incluída em ensaios de competição com outros cultivares, provou o seu bom comportamento agrícola, tendo superado os demais em produção. Contudo, não houve aceitação do produto pelo público consumidor, visto apresentar tipo de grão de qualidade e aspecto inferiores.

Os dados comparativos entre o cultivar IAC-435 (local), mais plantado, e o IR8, foram os seguintes:

\section{Cultivares}

IR8

IAC-435

Germinação

$10 / 10 / 69$

$10 / 10 / 69$

$6 / 11 / 69$

$6 / 11 / 69$

Florescimento

$7 / 2 / 70$

$27 / 2 / 70$

$6 / 2 / 70$

$1 / 4 / 70$

$25 / 2 / 70$

Colheita

$12 / 4 / 70$

$23 / 3 / 70$

Produção (kg/ha)

7.677

$2 / 4 / 70$

6.806

6.080

Produção (\%)

112,80

5.500

100,00

110,50

Cariopse - comp. (mm)

6,20

100,00

Cariopse - larg. (mm)

2,59

7,20

2,40

Em 1969, o Instituto Agronômico recebeu do Centro Internacional de Agricultura Tropical, CIAT, 71 amostras de novas progênies IR, descendentes do IR8. 
Com a finalidade de obter dados preliminares, esses cultivares foram plantados, sob condições irrigadas, na várzea do Centro Experimental de Campinas.

Após a colheita, foram escolhidos os seis melhores, segundo o tipo de grão, o porte e a produção, para avaliação das características agronômicas e tecnológicas.

Os resultados das comparações realizadas são apresentados nesta publicação.

\section{2 - MATERIAL E METODO}

Com base nos resultados, os seis cultivares escolhidos foram confrontados com o IAC-435 e o IAC-120, em experimento instalado no ano agrícola 1970-71 na várzea do Centro Experimental de Campinas, o qual obedeceu ao delineamento estatístico de blocos ao acaso, com seis repetições (3).

Cada canteiro do experimento compunha-se de cinco linhas de $5 \mathrm{~m}$ de comprimento, distanciadas de $0,30 \mathrm{~m}$ entre si, semeadas na base de 60 sementes por metro de sulco.

Aplicou-se no plantio uma adubação básica NPK de 4-60-30 $\mathrm{kg} / \mathrm{ha} \mathrm{e}$, em cobertura, $55 \mathrm{~kg} / \mathrm{ha}$ de $\mathrm{N}$, aos 40 dias após a germinação do arroz.

Os tratos culturais limitaram-se a capinas manuais, quando necessárias.

Foram anotados os dados sobre as características agronômicas e as observações sobre doenças (5).

$\mathrm{Na}$ avaliação sensorial, tanto para o arroz cru como para o cozido, foi utilizado o método preconizado por Kramer (7), o "ranking test", por meio de um delineamento em blocos ao acaso.

A equipe de degustação, empregada nos testes, era constituída de 14 mulheres, donas de casa, todas com experiência em avaliação dessa natureza.

O preparo das amostras para análise sensorial foi feito por aquecimento elétrico, durante 20 minutos, em panelas de aço inoxidável e água destilada.

A proporção de água e arroz foi, em média, de 1:2, baseada no poder de absorção de água, determinada para cada amostra. ,

As provas foram realizadas em cabinas individuais, com temperatura controlada, onde cada degustadora recebia as amostras em pratos pretos, numerados, à temperatura de $65^{\circ} \mathrm{C}$. 
O ambiente da prova foi mantido escurecido, iluminado apenas por pequeno foco de luz vermelha, a fim de mascarar possíveis diferenças de coloração entre as amostras e evitar erros de apreciação.

Para o arroz cru, solicitou-se a cada membro da equipe que julgasse sua preferência pela aparência geral; para o cozido, pela aparência geral, pelas características comestíveis e pela preferência geral.

No primeiro estudo, foram testadas as amostras de grãos dos cultivares IAC-120, IAC-435, IR634-9-6-2, IR634-32-5-3, IR665 4-1-1 e IR665-4-5-5, os dois primeiros como testemunhas.

Num segundo estudo, foi testado apenas material de IR 665-4-1-1 e IR665-4-5-5, cultivares que tinham maiores possibilidades de futuramente serem utilizados para plantios comerciais, inclusive de IAC-435 e IAC-120, como testemunha. O método e o delineamento empregados foram os mesmos do estudo anterior.

As análises sensoriais (1) foram executadas em laboratório especializado, do Instituto de Tecnologia de Alimentos, em Campinas.

\section{3 - RESULTADOS E DISCUSSÃO \\ 3.1 - CARACTERISTICAS AGRONOMICAS}

Nos quadros 1 a 4 , são apresentados os resultados obtidos para as várias características agronômicas e tecnológicas dos cultivares testados.

O IR665-4-5-5 tem boa precocidade, porte baixo, comprimento da cariopse acima dos limites estabelecidos para grãos extralongos e largura que mais se aproxima da preferência do consumidor.

Outros cultivares que se destacaram, embora de porte mais alto e maior ciclo, foram IR634-32-2-5 e IR634-9-6-2.

O IR665-4-1-1 possui bom porte, boa precocidade, porém o tamanho da cariopse enquadra-o nos tipos longos.

Em relação à produção, as médias dos cultivares IR665-4-1-1 até IR634-1-1-1 não diferiram entre si, ao nível de 5\% (Tukey), assim como os cultivares de IR634-32-2-5 até IAC-120, ao mesmo nível de significância, conforme demonstrado no quadro 2 .

O cultivar IAC-435 foi muito atacado por pássaros e Piricularia oryzae Cav. 
SOUZA, GARRUTTI \& CHAIB

Out., 1972

QuAdro 1. - Características da planta e do grão de cultivares de arroz testados em cultura de várzea irrigada, no Centro Experimental de Campinas, em 1969/70

\begin{tabular}{|c|c|c|c|c|c|}
\hline \multirow{2}{*}{ Cultivar } & \multicolumn{3}{|c|}{ Característica da planta } & \multicolumn{2}{|c|}{ Tamanho da cariopse } \\
\hline & Ciclo (1) & Altura & $\begin{array}{l}\text { Paní- } \\
\text { cula (2) }\end{array}$ & $\begin{array}{l}\text { Compri- } \\
\text { mento }\end{array}$ & Largura \\
\hline & dias & $\mathrm{cm}$ & $\mathrm{cm}$ & $m m$ & $m m$ \\
\hline IR665-4-1-1 $\ldots$ & 84 & 57,9 & 19,6 & 6,72 & 2,08 \\
\hline IR665-4-5-5 $\ldots$ & 86 & 60,7 & 20,3 & 7,06 & 2,20 \\
\hline IR634-9-6-2 $\ldots$ & 117 & 78,7 & 21,8 & 7,06 & 1,98 \\
\hline IR634-32-5-3 . . & 117 & 78,9 & 22,2 & 7,27 & 2,17 \\
\hline IR634-32-2-5 $\ldots$ & 117 & 81,3 & 22,6 & 7,22 & 2,13 \\
\hline IR634-1-1-1 & 109 & 59,4 & 20,3 & 6,79 & 2,27 \\
\hline IAC-120 & 103 & 105,2 & 23,3 & 7,64 & 2,44 \\
\hline IAC-435 & 117 & 114,9 & 24,3 & 7,13 & 2,30 \\
\hline
\end{tabular}

Quadro 2. - Produção e peso de 1000 grãos dos cultivares de arroz mencionados no quadro anterior

\begin{tabular}{|c|c|c|c|c|}
\hline \multirow[t]{2}{*}{ Amostra } & \multicolumn{2}{|c|}{ Produção relativa } & \multicolumn{2}{|c|}{ Peso de 1000 grãos } \\
\hline & $\mathrm{kg} / \mathrm{ha}$ & $\%$ & & $g$ \\
\hline IR665-4-1-1 & 7603 & 146,7 & & 25,44 \\
\hline IR665-4-5-5 $\ldots \ldots$ & 7510 & 144,9 & & 25,78 \\
\hline IR634-9-6-2 & 7384 & 142,5 & & 22,14 \\
\hline IR634-32-5-3 $\ldots$. & 7084 & 136,7 & & 27,40 \\
\hline IR634-32-2-5 & 6799 & 131,2 & & 25,62 \\
\hline IR634-1-1-1 & 6217 & 119,9 & & 23,60 \\
\hline IAC-120 $\ldots \ldots$ & 5181 & 100,0 & & 36,20 \\
\hline IAC-435 & 3484 & 67,2 & & 28,90 \\
\hline
\end{tabular}


Todos os cultivares IR foram superiores em produção ao IAC-435, diferindo deste, estatisticamente, ao nível de 5\%. Em relação ao IAC-120, apenas os quatro primeiros foram superiores a ele, estatisticamente, a esse nível.

As diferenças verificadas no peso de 1000 grãos foram resultantes do seu tamanho.

\section{2 - AVALIAÇAO SENSORIAL DO ARROZ CRU}

\subsection{1 - ESTUDO 1}

QuAdro 3. - Preferência pela aparência geral de alguns cultivares IAC e IR, para o arroz cru

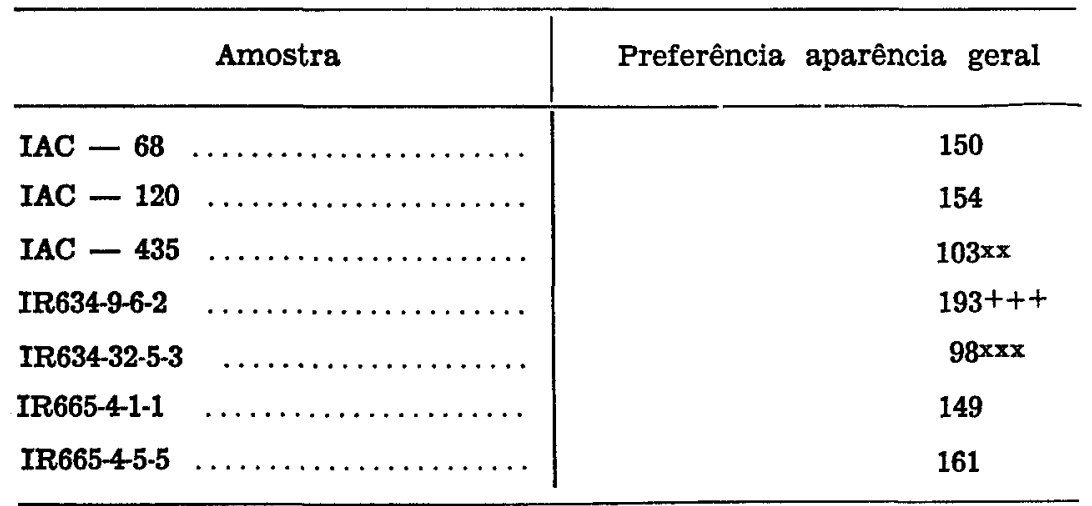

\footnotetext{
36 fulgamentos (114-173) $5 \%$; (108-79) $1 \%$; (101-186) $0,1 \%$

xx preferência ao nivel de $1 \%$

$\mathbf{x x x}$ preferencia ao nivel de $0,1 \%$

+++ rejelção ao nvel de $0,1 \%$
}

As amostras IR634-32-5-3 e IAC-435 foram preferidas aos níveis de $0,1 \%$ e $1 \%$, respectivamente. A amostra IR634-9-6-2 foi rejeitada ao nível de $0,1 \%$.

\subsection{2 - ESTUDO 2}

QuAdro 4. - Preferência pela aparência geral de alguns cultivares IAC e IR, para o arroz cru

\begin{tabular}{|c|c|c|c|}
\hline & Amostra & Preferência aparência & geral \\
\hline $\mathrm{IAC}-120$ & $\cdots \cdots$ & & 53 \\
\hline IAC -435 & $\ldots \ldots \ldots \ldots \ldots \ldots$ & & 58 \\
\hline IR665-4-1-1 & & & $72+$ \\
\hline IR665-4-5-5 & $\ldots \ldots \ldots \ldots \ldots \ldots \ldots$ & & 67 \\
\hline
\end{tabular}


A amostra do cultivar IR665-4-1-1 foi rejeitada ao nível de $5 \%$. Não houve diferenças significativas entre as demais amostras.

\section{3 - AVALIAÇÃO SENSORIAL DO ARROZ COZIDO}

\subsection{1 - ESTUDO 1}

QuADro 5. - Preferência pela aparência geral, características comestíveis e preferência geral de alguns cultivares IAC e IR, para arroz cozido

\begin{tabular}{|c|c|c|c|}
\hline \multirow[b]{2}{*}{ Amostra } & \multicolumn{3}{|c|}{ Preferência } \\
\hline & $\begin{array}{c}\text { Aparência } \\
\text { geral }\end{array}$ & $\begin{array}{c}\text { Característica } \\
\text { comestível }\end{array}$ & $\begin{array}{c}\text { Preferência } \\
\text { geral }\end{array}$ \\
\hline IAC-68 $\ldots \ldots \ldots$ & 108 & 106 & 108 \\
\hline IAC-120 $\ldots \ldots$ & $52 \times x \times$ & $54 \mathrm{xxx}$ & $54 \times x x$ \\
\hline IAC-435 $\ldots \ldots$ & 118 & 119 & 118 \\
\hline IR634-9-6-2 $\ldots \ldots$ & 121 & 120 & 120 \\
\hline IR634-32-5-3 $\ldots$ & $155^{+++}$ & $150+++$ & $151+++$ \\
\hline IR665-4-1-1 $\ldots$ & 110 & 109 & 101 \\
\hline IR665-4-5-5 $\ldots$ & 92 & 102 & 103 \\
\hline
\end{tabular}

27 julgamentos (82-133) $5 \%$; (77-138) $1 \%$; (71-144) $0,1 \%$ xxx preferência ao nivel de $0,1 \%$

+++ rejeição ao nível de $0,1 \%$

A amostra do cultivar IAC-120 foi a mais preferida ao nível de $0,1 \%$, na aparência geral, nas características comestíveis e na preferência geral, ao passo que a amostra do cultivar IR634$-32-5-3$ foi rejeitada em todas as características estudadas. Não houve diferença significativa de preferência, entre as demais.

Os resultados da análise sensorial de aparência geral para o cultivar IR634-32-5-3, em relação a arroz cru e arroz cozido, foram inversos, confirmando as observações das donas de casa e do público consumidor em geral.

\section{$3.3 .2-$ ESTUDO 2}

As amostras dos cultivares IR665-4-5-5 e IAC-120 foram as mais preferidas; a primeira, ao nível de $1 \%$, em relação à aparência geral e preferência geral, e a $5 \%$, para as características comestíveis; a segunda, ao nível de 5\%, para as três características. A 
Quadro 6. - Preferência pela aparência geral, características comestíveis e preferência geral de alguns cultivares IAC e IR, para arroz cozido

\begin{tabular}{|c|c|c|c|}
\hline \multirow{2}{*}{ Amostra } & \multicolumn{3}{|c|}{ Preferência } \\
\hline & $\begin{array}{c}\text { Aparência } \\
\text { geral }\end{array}$ & $\begin{array}{c}\text { Característica } \\
\text { comestivel }\end{array}$ & $\begin{array}{c}\text { Preferência } \\
\text { geral }\end{array}$ \\
\hline IAC- 120 & $38 x$ & $38 x$ & $38 x$ \\
\hline IAC-435 $\ldots \ldots$ & $71+$ & $68+$ & $73+$ \\
\hline IR665-4-1-1 $\ldots$ & 56 & 54 & 57 \\
\hline IR665-4-5-5 & $35 \times x$ & $38 x$ & $36 \times x \times$ \\
\hline
\end{tabular}

20 julgamentos (39-61) 5\%; (36-64) $1 \%$

$\mathrm{xx}$ preferência ao nivel de $1 \%$

+ rejeição ao nível de $5 \%$

$x$ preferência ao nível de $5 \%$

amostra do cultivar IAC-435, neste segundo estudo, foi rejeitada ao nível de $5 \%$, o que não aconteceu no primeiro estudo, em que havia um cultivar que lhe era inferior, como é o caso do IR634-32-5-3. A ordem de preferência foi: IR665-4-5-5, IAC-120, IR665-4-1-1 e IAC-435.

\section{4 - CONCLUSÕES}

Os resultados obtidos neste trabalho permitem tirar as seguintes conclusões:

1. Os cultivares IR são mais produtivos do que aqueles atualmente cultivados e recomendados pelo Instituto Agronômico de Campinas (10) .

2. O cultivar IR665-4-5-5, pelas suas características agronômicas e tecnológicas, é o que mais se aproxima das exigências do comércio interno dos estados centrais do Brasil.

3. Deverão ser continuadas as pesquisas no sentido de realizar estudos de cruzamento, a fim de conhecer a transmissão de suas boas características para os cultivares locais.

4. Deverão ser testados novos métodos de cozimento e de avaliação sensorial, além da determinação da composição química, para uma conclusão mais definitiva sobre os resultados obtidos. 
SOUZA, GARRUTTI \& CHAIB

\section{NEW CULTIVARS FOR IRRIGATED RICE CULTURE}

\section{SUMMARY}

The cultivars planted in the central states of Brazil, generally have extra long seeds, and the caryopsis is longer than $7 \mathrm{~mm}$ and wider than $2,4 \mathrm{~mm}$.

The IR8 cultivar is not accepted by public because it presents a type of seed of inferior quality and appearance. New IR cultivars were introduced and comparatively tested with the ones cultivated in the State of São Paulo - Brazil (IAC-120 and IAC-435).

The agronomical and technological characteristics (sensorial analyses) of the tested cultivars were evaluated.

Considering the relative production, IR665-4-1-1, IR665-4-5-5, IR634-9-6-2 and IR634-32-5-5 were superior to IAC-120 and IAC-435 by more than $30 \%$.

The cultivars IR634-32-5-3 and IAC-435 were the most preferred on the basis of sensorial analyses of raw rice, IR634-9-6-2 being rejected on $0.1 \%$ level.

For the cooked rice the most preferred were IAC-120 and IR665-4-5-5: IR634-32-5-3 was rejected on the $0.1 \%$ level by the testing panel on general appearance, eating quality and general preference.

Comparing all the agronomical characteristics (plant size, cycle, caryopsis size and production) and technological characteristics (general appearance and eating quality) of the processed and cooked rice, the IR665-4-5-5 cultivar is one that approximates most to the characteristics required by the farmers and the consumers in the Central States of Brazil.

\section{LITERATURA CITADA}

1. AMMERINE, N. A. et alii. Principles of sensory evaluation of food. New York, Academic Press, 196!. 300p.

2. BRASIL. Conselho Nacional do Comércio Exterior. Padronização do arroz. Lavoura Arrozeira, Porto Alegre, 24(260):3-13, 1971.

3. COCHRAN, W. G. \& COX, G. Experimental desigs. 2.ed. New York, John Wiley, $1964.611 \mathrm{p}$.

4. GERMEK, E. B. Métodos de cultura e variedades de arroz. Revista de Agricultura 21(7/8):264-270, 1946.

5. INTERNATIONAL RICE RESEARCH INSTITUTE. Catalog of rice cultivars and breeding lines (Oryza sativa $\mathrm{L}_{\text {.) }}$ in the world collection of the International Rice Research Institute. Los Baños, Philippines, 1970. 281p.

6. IRRI REPORTER, Filipinas, v.2, n. ${ }^{\circ}$, Sept. 1966.

7. KRAMER, A. A quick rank test for significance of differences in multiple comparisons. Food Techonology 8(10):391-392, 1956.

8. MIRANDA, H. S. Súmula dos trabalhos de arroz realizados em 1933 a 1948. Campinas, Instituto Agronômico, 1933/48.

9. SILVA, M. V. Possibilidades de melhoramento do arroz a partir da introdução de formas cultivadas do estrangeiro. Lisboa, Ministério da Economia, 1952. 19p.

10. SOUZA, D. M. Instruções para a cultura de arroz. Campinas, Instituto Agronômico, 1970. 12p. (Boletim 141) 